\title{
Regional ventilation distribution in healthy lungs: can reference values be established for electrical impedance tomography parameters?
}

\author{
Lin Yang ${ }^{1 \#}$, Meng Dai ${ }^{2 \#}$, Xinsheng Cao ${ }^{1}$, Knut Möller ${ }^{3}$, Mantas Dargvainis ${ }^{4}$, Inéz Frerichs ${ }^{4}$, \\ Tobias Becher ${ }^{4}$, Feng Fu ${ }^{2}$, Zhanqi Zhao ${ }^{2,3}$ \\ ${ }^{1}$ Department of Aerospace Medicine, Fourth Military Medical University, Xi'an, China; ${ }^{2}$ Department of Biomedical Engineering, Fourth Military \\ Medical University, Xi'an, China; ${ }^{3}$ Institute of Technical Medicine, Furtwangen University, Villingen-Schwenningen, Germany; ${ }^{4}$ Department of \\ Anaesthesiology and Intensive Care Medicine, University Medical Centre of Schleswig-Holstein Campus Kiel, Kiel, Germany \\ Contributions: (I) Conception and design: Z Zhao, K Möller, M Dargvainis, I Frerichs; (II) Administrative support: F. Fu; (III) Provision of study \\ materials or patients: M Dai, F Fu; (IV) Collection and assembly of data: L Yang, M Dai, Z Zhao; (V) Data analysis and interpretation: Z Zhao; (VI) \\ Manuscript writing: All authors; (VII) Final approval of manuscript: All authors. \\ \#These authors contributed equally to this work. \\ Correspondence to: Feng Fu; Zhanqi Zhao. Department of Biomedical Engineering, Fourth Military Medical University, No. 169 Changle West Road, \\ Xi'an 710032, China. Email: fengfu@fmmu.edu.cn; zhanqi.zhao@hs-furtwangen.de.
}

Background: Although electrical impedance tomography (EIT) is widely used for monitoring regional ventilation distribution, reference values have yet to be established for clinical use. The present study aimed to evaluate the feasibility of creating reference values for standard EIT parameters for potential clinical application.

Methods: A total of 75 participants with healthy lungs were included in this prospective study (male:female, 48:27; age, $34 \pm 14$ years; height, $172 \pm 7 \mathrm{~cm}$; weight, $73 \pm 12 \mathrm{~kg}$ ). The subjects were examined during spontaneous breathing in the supine position. EIT measurements were performed at the level of the $4^{\text {th }}$ intercostal space. Commonly used EIT-based parameters, including the center of ventilation (CoV), dorsal and most dorsal fractions of ventilation distribution $\left(T V_{D}\right.$ and $T V_{R O I 4}$ respectively), global inhomogeneity (GI) index, and standard deviation of regional ventilation delay index $\left(\mathrm{RVD}_{\mathrm{SD}}\right)$ were calculated.

Results: Following outlier detection, EIT data from 71 subjects were finally evaluated. The values of the evaluated parameters were: $\mathrm{CoV}, 48.7 \% \pm 1.7 \%$; TVD, $48.1 \% \pm 5.4 \%$; TV $\mathrm{ROI}, 7.1 \% \pm 1.8 \%$; GI, $0.49 \pm 0.04$; and $\mathrm{RVD}_{\mathrm{SD}}, 7.0 \pm 2.0$. The coefficients of variation for $\mathrm{CoV}$ and $\mathrm{GI}$ were low $(0.03$ and 0.07 , respectively), but those for $\mathrm{TV}_{\mathrm{ROI}}$ and $\mathrm{RVD}_{\mathrm{SD}}$ were comparatively high (0.26 and 0.28 , respectively). None of the evaluated parameters showed a significant correlation with age. The GI index showed a weak but significant correlation with body mass index $(\mathrm{R}=0.29, \mathrm{P}=0.01)$. The $\mathrm{RVD}_{\mathrm{SD}}$ was slightly higher in males than in females.

Conclusions: Our study indicated that $\mathrm{CoV}$ and GI were stable parameters with small coefficients of variation in participants with healthy lungs. The creation of EIT parameter reference values for setting treatment targets may be feasible.

Keywords: Electrical impedance tomography (EIT); regional ventilation distribution; standard EIT parameters

Submitted Nov 13, 2020. Accepted for publication Jan 24, 2021.

doi: $10.21037 /$ atm-20-7442

View this article at: http://dx.doi.org/10.21037/atm-20-7442 


\section{Introduction}

Chest electrical impedance tomography (EIT) is a noninvasive bedside imaging tool that is used to visualize regional lung ventilation and perfusion (1). EIT typically involves 16 or 32 electrodes being attached to the thorax's surface, through which imperceptible high-frequency electrical currents are delivered. The resulting voltages and reconstructed regional impedance values reflect the volume changes of air and blood in the lung tissue (2). Chest EIT applications cover a broad field of clinical practice (3), including the treatment of acute respiratory distress syndrome (ARDS) (4), coronavirus disease 2019 (5-7), and acute exacerbation of chronic obstructive pulmonary disease under mechanical ventilation $(8,9)$, as well as pulmonary function testing for patients with asthma, cystic fibrosis, and chronic obstructive pulmonary disease (10-13).

EIT images contain multiple physiological and pathophysiological processes that require further data processing depending on the intended clinical application. One of the main purposes of using EIT in clinical practice is to monitor gravity-dependent ventilation distribution during treatment (14-16). For instance, in ARDS patients, the ventilation distribution in the ventrodorsal direction (corresponding to the gravity-dependent direction in the supine or prone position) changes after the adjustment of positive end-expiratory pressure (PEEP), which may suggest recruitment or derecruitment (17). Several EITbased measures have been proposed to quantify the ventilation distribution in the ventrodorsal direction, including the center of ventilation $(\mathrm{CoV})$, a dorsal fraction of ventilation, and anterior-to-posterior ventilation ratio. A consensus statement was published to unify the terminology and definitions of EIT parameters (2) to support their unambiguous use and report. Previous studies have achieved equal distribution between ventral and dorsal regions as the target of treatment $(14,17,18)$, however, whether equal distribution is the ideal status is not validated. So far, no study has analyzed the ventrodorsal distribution systematically in a large study population. In a previous study of 13 healthy adults, Schibler et al. found that the mean value of $\mathrm{CoV}$ was centrally located but showed extremely high variation among participants $(50.5 \% \pm 14.7 \%)(19)$. Since EIT's rationale is to develop individualized ventilation strategies, many questions regarding EIT-based measures still need to be answered. These questions include whether normal ranges for these measures exist and whether the treatment target should be set according to one predefined $\mathrm{CoV}$ value, for instance, or be individualized. Similar questions need to be answered for other EITbased parameters, such as the global inhomogeneity (GI) index (20) and regional ventilation delay (RVD) (21), to assess the spatial and temporal ventilation distribution. The GI and RVD were proposed to guide PEEP optimization $(4,22)$ and weaning from ventilation (23). Standard reference values for these parameters may aid in the clinical application of EIT.

This preliminary study aimed to evaluate the spatial and temporal ventilation distribution in healthy lungs during spontaneous breathing in the supine position. The variations and the correlations with age and body mass index (BMI) were also examined. We present the following article in accordance with the MDAR reporting checklist (available at: http://dx.doi.org/10.21037/atm-20-7442).

\section{Methods}

\section{Subjects and measurement}

A total of 75 volunteers with healthy lungs (male:female, 48:27; age, $34 \pm 14$ years; height, $172 \pm 7 \mathrm{~cm}$; weight, $73 \pm 12 \mathrm{~kg}$ ) were prospectively examined by EIT. Eligible participants had no known lung diseases or contraindications for EIT. EIT was performed with the participants spontaneously breathing in the supine position. An EIT electrode belt with 16 electrodes was placed around the thorax at the $4^{\text {th }}$ intercostal space, and 1 reference electrode was placed at the abdomen (PulmoVista 500, Dräger Medical, Lübeck, Germany). In female subjects, if the electrode belt could not be placed at the designated level due to the breasts' position, the belt was placed slightly above. Electrical alternating currents were applied in a sequential rotating process. The frequency and amplitude of the currents were determined automatically according to the measurement environment's background noise. The resulting surface potential differences between neighboring electrode pairs were measured and recorded at $20 \mathrm{~Hz}$. Each EIT measurement session lasted 2 minutes.

\section{EIT data analysis}

The finite element method (FEM)-based, linearized Newton-Raphson reconstruction algorithm of Pulmovista 500 (Dräger Medical, Lübeck, Germany) was used to convert the recorded data into EIT images (24). The exact algorithm is confidential and unpublished. Based on the description (24), the algorithm calculates the sensitivity 


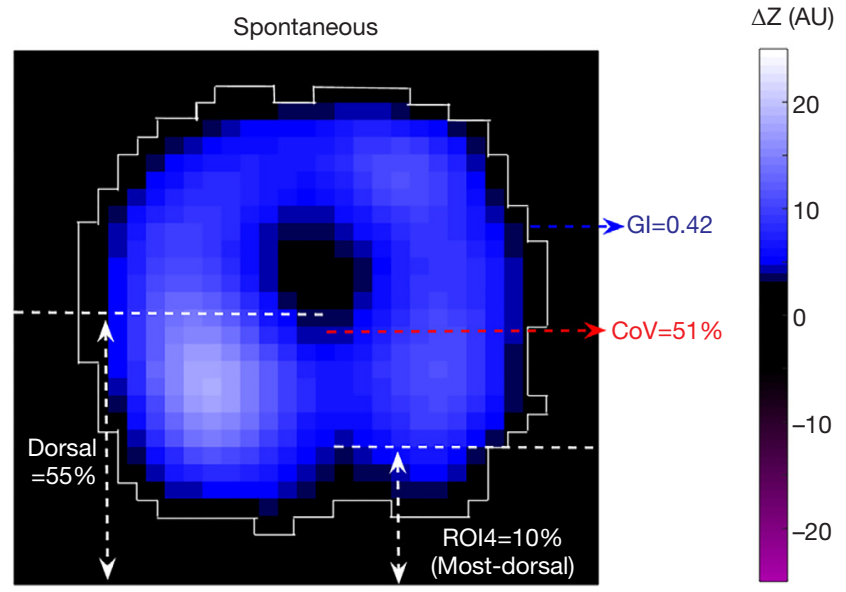

Figure 1 Illustration of EIT-based parameters in a tidal variation image of an individual with healthy lungs during spontaneous breathing. Regional ventilation delay can only be assessed with a series of EIT-images and is therefore not included in this illustration. EIT, electrical impedance tomography; GI, the global inhomogeneity index; $\mathrm{CoV}$, center of ventilation; ROI 4 , the $4^{\text {th }}$ region of interest; $\Delta \mathrm{Z}$, relative impedance changes; $\mathrm{AU}$, arbitrary unit.

matrix for an FEM with 340 triangular elements. A Gaussian filter is used to smooth the reconstructed image, and bilinear interpolation is applied to increase the virtual resolution. Image reconstruction using this algorithm was achieved with the manufacturer's software (EIT Data Review Tool, Dräger Medical, Lübeck, Germany).

Functional EIT (fEIT)-tidal variation (TV) was calculated by subtracting the end-expiration from the endinspiration image, representing the variation during tidal breathing. Tidal images of 1 minute were averaged to increase the signal-to-noise ratio.

$$
T V_{i}=\frac{1}{N} \sum_{n=1}^{N}\left(\Delta Z_{i, I n s, n}-\Delta Z_{i, E x p, n}\right)
$$

in which $T V_{i}$ is the pixel $i$ in the fEIT image; $N$ is the number of breaths within the analyzed period; and $\Delta Z_{i, I n s \text {, }}$ and $\Delta Z_{i, E x p}$ are the pixel values in the raw EIT image at endinspiration and end-expiration, respectively. When $T V_{i}<0$, a value of 0 was assigned to $T V_{i}$.

$\mathrm{Co} V$ depicts ventilation distribution influenced by gravity or various lung diseases (relative impedance value weighted with a location in the anteroposterior coordinate) (25):

$$
\mathrm{CoV}=\sum\left(y_{i} \times T V_{i}\right) / \sum T V_{i} \times 100 \%
$$

in which $T V_{i}$ is the impedance change in the fEIT image for pixel $i ; y_{i}$ is the height of pixel $i$, and the value is scaled so that the bottom of the image (dorsal) is $100 \%$ and the top (ventral) is $0 \%$.

The dorsal fraction of ventilation is calculated as the sum of all pixel values in the dorsal half of the fEIT image over the sum of all pixel values from the fEIT image (Figure 1):

$$
T V_{D}=\sum_{j=513}^{1024} T V_{j} / \sum_{i=1}^{1024} T V_{i} \times 100 \%
$$

The anterior-to-posterior ventilation ratio can be deduced from $T V_{D}$ as $\left(1-T V_{D}\right) / T V_{D}$, and it was not calculated separately in the present study.

The most dorsal region is usually denoted as the $4^{\text {th }}$ region of interest (ROI4) after the division of the image into 4 horizontal, anterior to posterior segments of equal height. The ventilation distributed in these regions is calculated as follows:

$$
T V_{R O I 4}=\sum_{j=769}^{1024} T V_{i} / \sum_{i=1}^{1024} T V_{i} \times 100 \%
$$

The $G I$ index is calculated from the tidal EIT images to summarize the heterogeneity of ventilation (26).

$$
G I=\sum_{l \in \text { lung }}\left|T V_{l}-\operatorname{median}\left(T V_{\text {lung }}\right)\right| / \sum_{l \in \text { lung }} T V_{l}
$$

in which $T V$ denotes the value of the differential impedance in the tidal images; $T V_{l}$ is the pixel in the identified lung area; pixel $l$ is considered as a lung region if $T V_{l}>10 \% \times \max (T V) . T V_{\text {lung }}$ denotes all pixels representing the lung area. A high $G I$ index implies high variation among pixel tidal impedance values.

The RVD index characterizes the regional ventilation delay as pixel impedance rising time compared to the global impedance curve (21), which may be used to assess tidal recruitment/derecruitment.

$$
R V D_{l}=t_{l, 40 \%} / T_{\text {inspiration, global }} \times 100 \%
$$

in which $t_{l, 40 \%}$ is the time needed for pixel $l$ to reach $40 \%$ of its maximum inspiratory impedance change. $T_{\text {inspiration, }}$ global denotes the inspiration time calculated from the global impedance curve. For assessment of the distribution of RVD, Muders et al. proposed the use of the standard deviation of the pixel values (21):

$$
R V D_{S D}=\sqrt{\frac{1}{L} \sum_{l \in \text { lung }}\left(R V D_{l}-\text { mean }\left(R V D_{\text {lung }}\right)\right)^{2}}
$$

in which $\mathrm{L}$ is the total number of pixels identified as the lung area.

\section{Statistical analysis}

Data analyses were performed using MATLAB 8.5 (The MathWorks Inc., Natick, USA). The Lilliefors test was used to test for normality. For normally distributed data, 
Table 1 EIT-based parameters and their correlations with age and BMI

\begin{tabular}{lcccc}
\hline EIT parameters $(\mathrm{n}=71)$ & Mean $\pm \mathrm{SD}$ & Coefficient of variation & Correlation with age $(\mathrm{R}, \mathrm{P})$ & Correlation with BMI $(\mathrm{R}, \mathrm{P})$ \\
\hline $\mathrm{CoV}(\%)$ & $48.7 \pm 1.7$ & 0.03 & $-0.03,0.82$ & $0.10,0.41$ \\
$\operatorname{TV}_{\mathrm{D}}(\%)$ & $48.1 \pm 5.4$ & 0.11 & $0.01,0.91$ & $0.03,0.78$ \\
$\mathrm{TV}_{\mathrm{ROI4}}(\%)$ & $7.1 \pm 1.8$ & 0.26 & $0.07,0.55$ & $-0.08,0.51$ \\
$\mathrm{Gl}$ & $0.49 \pm 0.04$ & 0.07 & $0.22,0.07$ & $0.29,0.01^{*}$ \\
$\mathrm{RVD}_{\mathrm{SD}}$ & $7.0 \pm 2.0$ & 0.28 & $-0.14,0.25$ & $0.04,0.73$ \\
\hline
\end{tabular}

${ }^{*} \mathrm{P}<0.05$. EIT, electrical impedance tomography; CoV, center of ventilation; $\mathrm{TV}_{\mathrm{D}}$, dorsal fraction of ventilation; $\mathrm{TV}_{\mathrm{ROI}}$, ventilation distribution in the most dependent region; Gl, the global inhomogeneity index; $\mathrm{RVD}_{\mathrm{SD}}$, standard deviation of the regional ventilation delay index; $\mathrm{R}$, correlation coefficient; BMI, body mass index.

results were expressed as means \pm standard deviations. Outliers of each EIT-based parameter were analyzed using the modified Z-score. Subject $\mathrm{k}$ was defined as an outlier if $\left|z_{k}\right|>3.5$. The coefficient of variation was calculated after the removal of outliers (for EIT-based parameters only). The correlations of the parameters with age and BMI were evaluated using Pearson's linear correlation. The values of male and female subjects were compared using boxplots. $C o V$ and $T V_{D}$ were compared by paired $t$-test. A $\mathrm{P}$ value $<0.05$ was considered to be statistically significant.

\section{Ethical approval}

The study was conducted following the Declaration of Helsinki (as revised in 2013). The study protocol was approved by the ethics committees of the Fourth Military Medical University (KY202004769-5) and University Medical Centre of Schleswig-Holstein Campus Kiel (D426/19). Written informed consent was obtained from all subjects before the study. This study was carried out following relevant regulations and guidelines.

\section{Results}

EIT measurement was successfully performed in 75 participants. In total, 4 outliers were detected and excluded from the final analysis (1 subject for each parameter except $\left.T V_{R O I}\right)$. The evaluated parameters are summarized in Table 1. The coefficients of variation for $\mathrm{CoV}$ and GI were low, but those for $\mathrm{TV}_{\mathrm{ROI}}$ and $\mathrm{RVD}_{\mathrm{SD}}$ were extremely high. The GI index showed a weak but significant correlation with $\mathrm{BMI}(\mathrm{R}=0.29, \mathrm{P}=0.01)$.

In Figure 2, the parameters are plotted against age or BMI. Data of male and female participants are marked with different colors. Three participants who had a modified
Z-score of BMI $>3.5$ were female (not excluded). The values of male $(n=45)$ and female $(n=26)$ participants are compared in a boxplot in Figure 3. The RVD $\mathrm{SD}_{\mathrm{SD}}$ was found to be slightly higher in males compared to females.

\section{Discussion}

In this preliminary study, 71 participants with healthy lungs underwent EIT examination, and multiple EIT-based parameters were evaluated. We found that $\mathrm{CoV}$ and GI were relatively stable, whereas $\mathrm{TV}_{\mathrm{ROI}}$ and $\mathrm{RVD}_{\mathrm{SD}}$ showed considerable variation among participants. The evaluated parameters were not correlated with age or BMI, except for the GI index, which had a weak correlation with BMI. The present study attempted to identify reference values that might be useful in the treatment of pulmonary diseases.

Both $\mathrm{TV}_{\mathrm{D}}$ and $\mathrm{CoV}$ reflect gravity-dependent influences on ventilation distribution. Clinical users sometimes confuse $\mathrm{TV}_{\mathrm{D}}$ with $\mathrm{CoV}$ due to the terminology (27). In the present study, we found $\mathrm{CoV}$ to have a considerably lower coefficient of variation than $\mathrm{TV}_{\mathrm{D}}$. This implies that $\mathrm{CoV}$ may be more stable and, therefore, might be superior to $T V_{D}$ in assessing whether the ventral-to-dorsal ventilation distribution of a patient is within the normal range (Table 1). A previous study showed that simple regions of interest might exhibit some variations, even in the same participant (28). Also, $\mathrm{TV}_{\mathrm{D}}$ cannot distinguish any regional distributions within the ventral or dorsal half of the functional EIT image, downgrading the spatial image resolution to $1 \times 2$. However, since $T V_{D}$ can be calculated easily, a previous study proposed its use for PEEP titration (17). For clinical applications, the user should consider that $\mathrm{TV}_{\mathrm{D}}$ has large variability even in healthy individuals during spontaneously breathing and, therefore, aiming to achieve a $\mathrm{TV}_{\mathrm{D}}$ of $50 \%$ may not be appropriate for 

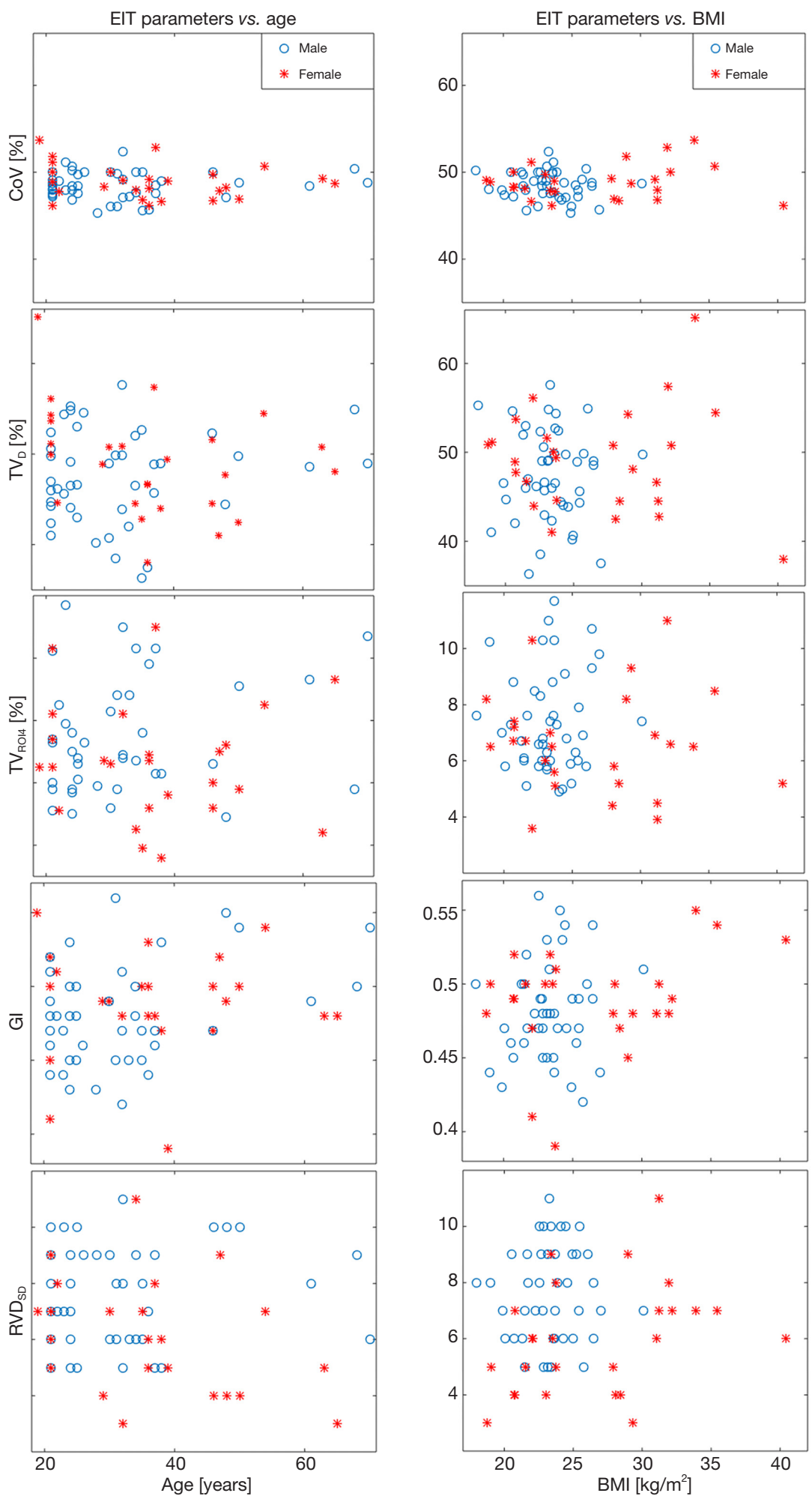

Figure 2 EIT-based parameters versus age and BMI, respectively. Blue circles for male subjects and red stars for female subjects. EIT, electrical impedance tomography; $\mathrm{CoV}$, center of ventilation; $\mathrm{TV}_{\mathrm{D}}$, dorsal fraction of ventilation; $\mathrm{TV}_{\mathrm{ROI}}$, ventilation distribution in the most dependent region; GI, the global inhomogeneity index; $\mathrm{RVD}_{\mathrm{SD}}$, the standard deviation of the regional ventilation delay index. 

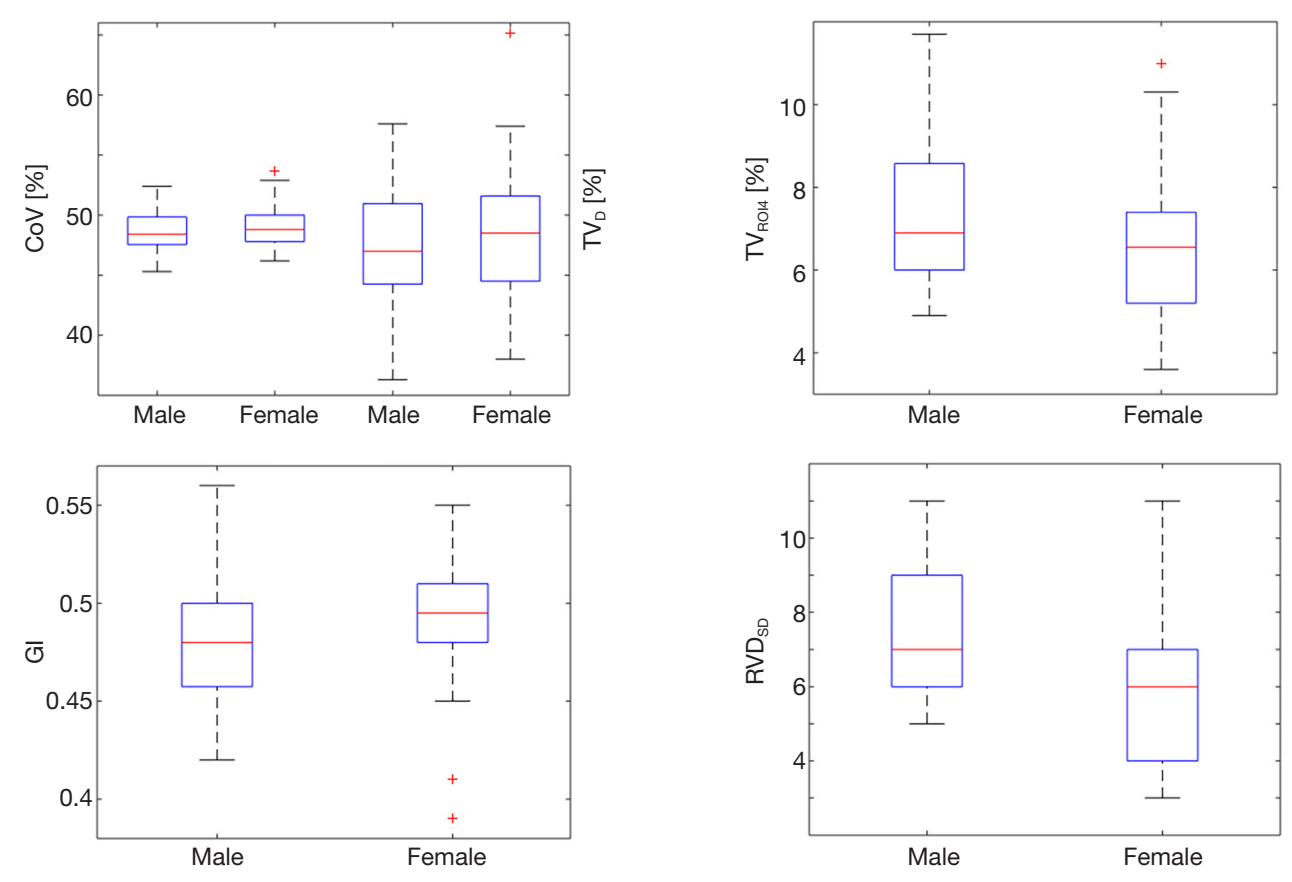

Figure 3 Boxplot of the EIT-based parameters of male and female subjects. The boxes mark the quartiles while the whiskers extend from the box out to the most extreme data values within 1.59 of the sample's interquartile range. EIT, electrical impedance tomography; CoV, center of ventilation; $\mathrm{TV}_{\mathrm{D}}$, dorsal fraction of ventilation; $\mathrm{TV}_{\mathrm{ROI}}$, ventilation distribution in the most dependent region; GI, the global inhomogeneity index; $\mathrm{RVD}_{\mathrm{SD}}$, the standard deviation of the regional ventilation delay index.

all patients (Figure 2).

The ventilation distribution in the most dependent region $\left(\mathrm{TV}_{\mathrm{ROI}}\right)$ was also used to assess the lung recruitment (29). The device manufacturer suggested the optimal value for $\mathrm{TV}_{\mathrm{ROI}}$ to be $10-15 \%$, which is not supported by the current findings (Table 1). We previously found that $\mathrm{TV}_{\mathrm{ROI}}$ may be influenced by spontaneous breathing or the presence of pendelluft (30-32). Although pendelluft is not common in healthy lungs, the diaphragmatic movement might have caused the high coefficient of variation found in this study. To confirm this hypothesis, $\mathrm{TV}_{\mathrm{ROI}}$ during forced inhalation or exhalation could be compared with resting tidal breathing, but such a comparison was not performed in this study. $\mathrm{TV}_{\mathrm{ROI}}$ should also be further investigated in lung-healthy patients under mechanical ventilation, with or without spontaneous breathing, under which conditions the diaphragmatic function would be completely different from that of the current study group.

The GI index is typically used to evaluate the ventilation heterogeneity within predefined lung regions, which is strongly dependent on lung area identification $(20,33)$. In individuals with healthy lungs, such as those investigated in the current study, alveolar collapse or overdistension is improbable. Therefore, simple lung area identification based on functional images of tidal variation would be sufficient (34). For diseased lungs, a recent study suggested that establishing a reference GI value might be possible by identifying standardized lung regions on individualized CT images (35). Pulletz et al. investigated different thresholds for identifying lung regions (36). A threshold of $20 \%$ has frequently been reported in the literature. Since the device manufacturer uses $10 \%$ to calculate RVD in the software, we also selected $10 \%$ to evaluate GI. For higher threshold values, the average GI values would become smaller.

The RVD parameter was validated in an in vivo study under the condition of a low-flow maneuver during mechanical ventilation, and a close correlation was observed between tidal recruitment and $\mathrm{RVD}_{\mathrm{SD}}$ (21). RVD measurement should also be conducted in the clinical setting when patients are ventilated under a lowflow maneuver (37). Muders et al. proposed reducing the volume during the low-flow maneuver to increase this method's applicability (38). The application of RVD under 
a normal spontaneous breathing pattern has also been reported in the literature (39); however, caution needs to be taken in interpreting the results. For our subject group with relatively uniform functional lung status, the coefficient of variation was large compared to other evaluated EIT parameters. With normal inspiration, the time taken is usually much shorter than the inspiration time during a low-flow maneuver, far fewer EIT data points are acquired, and there is a greater amount of noise. All of these factors may have contributed to the high level of variation observed. The sampling rate and signal filtering may impact the results, but they were not investigated in the current study.

None of the parameters were strongly correlated with age or BMI. Most of our participants were relatively young ( $<50$ years); thus, age dependency could not be well analyzed. Nevertheless, our finding supported the feasibility of establishing standard reference values for the EIT-based parameters. Although the electrode belt position differed slightly between males and females, the differences in the values of parameters between the sexes were insignificant. Previous studies have suggested that the impedance-volume ratio may be substantially altered when the electrode is positioned close to the abdomen $(40,41)$. Hence, the electrodes must be placed cranially to the breasts when performing EIT measurement on females, unless a particular application requires a specific placement (e.g., to observe diaphragmatic movement). One outlier was detected for each of the parameters except $T V_{\text {ROI4 }}$. As discussed, the calculations for the parameters are quite different. Therefore, certain outliers in data may influence 1 parameter but not the others. Nevertheless, we excluded 4 subjects to ensure data from the same number of participants were available to calculate the parameters.

We acknowledge that the present study has the following limitations. First, the number of participants was small. Second, only correlations of the parameters with age and BMI were examined. Furthermore, only 1 condition each was evaluated for breathing and body position. Third, as discussed above, the parameters' values can be influenced by many factors, which could not be entirely addressed in the present study. It should be noted that the reference values we attempted to establish were measured under the documented conditions using a specific EIT device, with a sampling rate of $20 \mathrm{~Hz}$, a low-pass filter of $0.83 \mathrm{~Hz}$ (corresponding to 50/minutes), a lung area threshold of $10 \%$, and the belt positioned at the $4^{\text {th }}$ intercostal space. Therefore, the results might not apply to other settings.
As a feasibility study, this work was only able to establish a framework for standardizing EIT-based reference parameters for clinical use. It would be interesting to explore the EIT parameters under various ventilation modes in lung-healthy patients in a future study.

\section{Conclusions}

Our study of individuals with healthy lungs indicated that the CoV and GI index were stable among the evaluated EIT-based parameters, with a small variation coefficient, whereas $\mathrm{SD}_{\mathrm{RVD}}$ and $\mathrm{TV}_{\mathrm{ROI}}$ were comparatively unstable. According to our results, standardizing the $\mathrm{CoV}$ and GI index's reference values for setting treatment targets could be feasible.

\section{Acknowledgments}

Funding: The study was partially supported by Everest Program of FMMU (2019ZFB002), Medical Program of FMMU (2018HKTS10 and 2019ZTC01), Equipment Program of PLA (KJ2018-2019C132), Postdoc Program (2019M663988), National Natural Science Foundation of China (51837011 and 61901478), BMBF MOVE (FKZ 13FH628IX6) and H2020 MCSA Rise \#872488-DCPM. The funding sources had no involvements in study design; collection, analysis, and interpretation of data; or writing of the report.

\section{Footnote}

Reporting Checklist: The authors have completed the MDAR reporting checklist. Available at http://dx.doi.org/10.21037/ atm-20-7442

Data Sharing Statement: Available at http://dx.doi. org/10.21037/atm-20-7442

Conflicts of Interest: All authors have completed the ICMJE uniform disclosure form (available at http://dx.doi. org/10.21037/atm-20-7442). ZZ received a consulting fee from Dräger Medical. Inéz Frerichs reports funding from the European Union's Framework Programme for Research and Innovation Horizon2020 (WELMO, Grant No. 825572; Projects CRADL, Grant No. 668259) and reimbursement of speaking fees, congress fees, and travel costs by Dräger Medical. Tobias Becher reports speaking fees and reimbursement of travel costs by Dräger Medical, 
Löwenstein Medical and Sedana Medical. The other authors have no conflicts of interest to declare.

Ethical Statement: The authors are accountable for all aspects of the work in ensuring that questions related to the accuracy or integrity of any part of the work are appropriately investigated and resolved. The study was conducted in accordance with the Declaration of Helsinki (as revised in 2013). The study protocol was approved by the ethics committees of the Fourth Military Medical University (KY202004769-5) and University Medical Centre of Schleswig-Holstein Campus Kiel (D426/19). Written informed consent was obtained from all participants prior to the study. This study was carried out in accordance with relevant regulations and guidelines.

Open Access Statement: This is an Open Access article distributed in accordance with the Creative Commons Attribution-NonCommercial-NoDerivs 4.0 International License (CC BY-NC-ND 4.0), which permits the noncommercial replication and distribution of the article with the strict proviso that no changes or edits are made and the original work is properly cited (including links to both the formal publication through the relevant DOI and the license). See: https://creativecommons.org/licenses/by-nc-nd/4.0/.

\section{References}

1. He H, Chi Y, Long Y, et al. Bedside Evaluation of Pulmonary Embolism by Saline Contrast Electrical Impedance Tomography Method: A Prospective Observational Study. Am J Respir Crit Care Med 2020;202:1464-8.

2. Frerichs I, Amato MB, van Kaam AH, et al. Chest electrical impedance tomography examination, data analysis, terminology, clinical use and recommendations: consensus statement of the TRanslational EIT developmeNt stuDy group. Thorax 2017;72:83-93.

3. Zhao Z, Fu F, Frerichs I. Thoracic electrical impedance tomography in Chinese hospitals: a review of clinical research and daily applications. Physiol Meas 2020;41:04TR1.

4. Zhao Z, Chang MY, Gow CH, et al. Positive end-expiratory pressure titration with electrical impedance tomography and pressure-volume curve in severe acute respiratory distress syndrome. Ann Intensive Care 2019;9:7.

5. Mauri T, Spinelli E, Scotti E, et al. Potential for Lung Recruitment and Ventilation-Perfusion Mismatch in Patients With the Acute Respiratory Distress Syndrome
From Coronavirus Disease 2019. Crit Care Med 2020;48:1129-34.

6. Sella N, Zarantonello F, Andreatta G, et al. Positive end-expiratory pressure titration in COVID-19 acute respiratory failure: electrical impedance tomography vs. PEEP/FiO2 tables. Crit Care 2020;24:540.

7. Fu Y, Zou R, Wang S, et al. Monitoring bronchoalveolar lavage with electrical impedance tomography: first experience in a patient with COVID-19. Physiol Meas 2020;41:085008.

8. Zhao Z, Chang MY, Frerichs I, et al. Regional air trapping in acute exacerbation of obstructive lung diseases measured with electrical impedance tomography: a feasibility study. Minerva Anestesiol 2020;86:172-80.

9. Frerichs I, Achtzehn U, Pechmann A, et al. Highfrequency oscillatory ventilation in patients with acute exacerbation of chronic obstructive pulmonary disease. J Crit Care 2012;27:172-81.

10. Zhao Z, Fischer R, Frerichs I, et al. Regional ventilation in cystic fibrosis measured by electrical impedance tomography. J Cyst Fibros 2012;11:412-8.

11. Munir B, Murphy EK, Mallick A, et al. A robust and novel electrical impedance metric of pulmonary function in ALS patients. Physiol Meas 2020;41:044005.

12. Vogt B, Zhao Z, Zabel P, et al. Regional lung response to bronchodilator reversibility testing determined by electrical impedance tomography in chronic obstructive pulmonary disease. Am J Physiol Lung Cell Mol Physiol 2016;311:L8-19.

13. Frerichs $I, Z$ hao $Z$, Becher T, et al. Regional lung function determined by electrical impedance tomography during bronchodilator reversibility testing in patients with asthma. Physiol Meas 2016;37:698-712.

14. Blankman P, Hasan D, van Mourik MS, et al. Ventilation distribution measured with EIT at varying levels of pressure support and Neurally Adjusted Ventilatory Assist in patients with ALI. Intensive Care Med 2013;39:1057-62.

15. Hough JL, Johnston L, Brauer SG, et al. Effect of body position on ventilation distribution in preterm infants on continuous positive airway pressure. Pediatr Crit Care Med 2012;13:446-51.

16. Lehmann S, Leonhardt S, Ngo C, et al. Electrical impedance tomography as possible guidance for individual positioning of patients with multiple lung injury. The clinical respiratory journal 2018;12:68-75.

17. Yoshida T, Piraino T, Lima CAS, et al. Regional Ventilation Displayed by Electrical Impedance Tomography as an Incentive to Decrease Positive End-Expiratory Pressure. Am J Respir Crit Care Med 2019;200:933-7. 
18. Karsten J, Krabbe K, Heinze H, et al. Bedside monitoring of ventilation distribution and alveolar inflammation in community-acquired pneumonia. J Clin Monit Comput 2014;28:403-8.

19. Schibler A, Yuill M, Parsley C, et al. Regional ventilation distribution in non-sedated spontaneously breathing newborns and adults is not different. Pediatr Pulmonol 2009;44:851-8.

20. Zhao Z, Möller K, Steinmann D, et al. Evaluation of an electrical impedance tomography-based global inhomogeneity index for pulmonary ventilation distribution. Intensive Care Med 2009;35:1900-6.

21. Muders T, Luepschen H, Zinserling J, et al. Tidal recruitment assessed by electrical impedance tomography and computed tomography in a porcine model of lung injury*. Crit Care Med 2012;40:903-11.

22. Girrbach F, Petroff D, Schulz S, et al. Individualised positive end-expiratory pressure guided by electrical impedance tomography for robot-assisted laparoscopic radical prostatectomy: a prospective, randomised controlled clinical trial. Br J Anaesth 2020;125:373-82.

23. Bickenbach J, Czaplik M, Polier M, et al. Electrical impedance tomography for predicting failure of spontaneous breathing trials in patients with prolonged weaning. Crit Care 2017;21:177.

24. Teschner E, Imhoff M. Electrical Impedance Tomography: The realization of regional ventilation monitoring. Lübeck, Germany: Dräger Medical GmbH; 2010.

25. Frerichs I, Hahn G, Golisch W, et al. Monitoring perioperative changes in distribution of pulmonary ventilation by functional electrical impedance tomography. Acta Anaesthesiol Scand 1998;42:721-6.

26. Zhao Z, Steinmann D, Frerichs I, et al. PEEP titration guided by ventilation homogeneity: a feasibility study using electrical impedance tomography. Crit Care 2010;14:R8.

27. Frerichs I, Zhao Z, Becher T. Simple Electrical Impedance Tomography Measures for the Assessment of Ventilation Distribution. Am J Respir Crit Care Med 2020;201:386-8.

28. Reifferscheid F, Elke G, Pulletz S, et al. Regional ventilation distribution determined by electrical impedance tomography: reproducibility and effects of posture and chest plane. Respirology 2011;16:523-31.

29. Wolf GK, Gomez-Laberge C, Rettig JS, et al. Mechanical ventilation guided by electrical impedance tomography in experimental acute lung injury. Crit Care Med 2013;41:1296-304.

30. Sun Q, Liu L, Pan C, et al. Effects of neurally adjusted ventilatory assist on air distribution and dead space in patients with acute exacerbation of chronic obstructive pulmonary disease. Crit Care 2017;21:126.

31. Zhao Z, Peng SY, Chang MY, et al. Spontaneous breathing trials after prolonged mechanical ventilation monitored by electrical impedance tomography: an observational study. Acta Anaesthesiol Scand 2017;61:1166-75.

32. Sang L, Zhao Z, Yun PJ, et al. Qualitative and quantitative assessment of pendelluft: a simple method based on electrical impedance tomography. Ann Transl Med 2020;8:1216.

33. Zhao Z, Steinmann D, Muller-Zivkovic D, et al. A lung area estimation method for analysis of ventilation inhomogeneity based on electrical impedance tomography. J Xray Sci Technol 2010;18:171-82.

34. Zhao Z, Yun PJ, Kuo YL, et al. Comparison of different functional EIT approaches to quantify tidal ventilation distribution. Physiol Meas 2018;39:01NT.

35. Yang L, Dai M, Moller K, et al. Lung regions identified with CT improve the value of global inhomogeneity index measured with electrical impedance tomography. Quant Imaging Med Surg 2021;11:1209-19.

36. Pulletz S, van Genderingen HR, Schmitz G, et al. Comparison of different methods to define regions of interest for evaluation of regional lung ventilation by EIT. Physiol Meas 2006;27:S115-27.

37. Haase J, Buchloh DC, Hammermuller S, et al. Mechanical Ventilation Strategies Targeting Different Magnitudes of Collapse and Tidal Recruitment in Porcine Acid Aspiration-Induced Lung Injury. J Clin Med 2019;8:1250.

38. Muders T, Hentze B, Simon P, et al. A Modified Method to Assess Tidal Recruitment by Electrical Impedance Tomography. J Clin Med 2019;8:1161.

39. Lumb AB, Savic L, Horsford MR, et al. Effects of tracheal intubation and tracheal tube position on regional lung ventilation: an observational study. Anaesthesia 2020;75:359-65.

40. Karsten J, Stueber T, Voigt N, et al. Influence of different electrode belt positions on electrical impedance tomography imaging of regional ventilation: a prospective observational study. Crit Care 2016;20:3.

41. Krueger-Ziolek S, Schullcke B, Kretschmer J, et al. Positioning of electrode plane systematically influences EIT imaging. Physiol Meas 2015;36:1109-18.

Cite this article as: Yang L, Dai M, Cao X, Möller K, Dargvainis M, Frerichs I, Becher T, Fu F, Zhao Z. Regional ventilation distribution in healthy lungs: can reference values be established for electrical impedance tomography parameters? Ann Transl Med 2021;9(9):789. doi: 10.21037/atm-20-7442 